\title{
Modélisation numérique de formes de fond périodiques en domaine peu profond. Exemple : les bancs de sable
}

\author{
Déborah Idier $^{1-2}$ et Dominique Astruc ${ }^{1}$
}

\author{
1. IMFT, Allée Pr. Camille Soula, 31400 Toulouse, France. 056128 \\ 5861.astruc@imft.fr. \\ 2. EPSHOM/CH/GG/Sédimentologie, 13, rue Le Chatellier, BP \\ 30316,29603Brest cedex,France.0298377660.idier@shom.fr.
}

Résumé

Un modèle morphodynamique 2DH est utilisé pour calculer les propriétés de stabilité d'un fond sableux soumis à un courant permanent. Les résultats sont validés par rapport à une analyse de stabilité linéaire et montrent la génération de bancs de sable. Le modèle permet de déterminer la hauteur de saturation du banc. A partir du taux de croissance et de la hauteur de saturation calculés par le modèle, une équation d'amplitude est utilisée pour calculer l'évolution temporelle de l'amplitude du banc, jusqu'à sa saturation. Les résultats sont cohérents avec les observations in-situ.

\section{Abstract}

A $2 \mathrm{DH}$ morphodynamical model is used to compute the stability properties of a sandy bottom subjected to a quasi-steady current. The results are in good agreement with linear stability analysis results and feature the generation of sandbanks. The numerical model is used to estimate the fully-developped sandbank height. Then, using the predicted growth rate and the fully-developped height, an amplitude equation is used to predict the evolution of the sandbank until the saturation. The results are in agreement with field data.

\section{Introduction}

Les bancs ou dunes de sable tidales sont des structures de grande échelle très courantes sur le plateau continental, caractérisées par des longueurs d'onde supérieures à la hauteur d'eau. La migration de certaines dunes peut dépasser 150 $m$ par an [1]. Cependant, bien qu'elles présentent une grande importance pratique (sécurité de la navigation [2], pipelines, dragages, ...), leur morphodynamique n'est pas encore complètement comprise. Des études de terrain [2] ont été réalisées, mais fournissent des données limitées en temps et en espace. Une autre approche est l'analyse de stabilité linéaire [3] ou faiblement non-linéaire [4], qui permet de calculer le taux de croissance d'une perturbation bathymétrique d'amplitude infinitésimale. Hulscher [3] montre qu'avec une description hydrodynamique $2 \mathrm{DH}$, seuls des bancs de sable peuvent être générés. Une description 3D permet cependant de générer des dunes [3]. 
Afin de prendre en compte les processus non-linéaires, non négligeables à amplitude finie, le problème peut être appréhendé à l'aide de la modélisation numérique. Bien que les modèles morphodynamiques soient couramment utilisés en ingénierie côtière (calculs de flux de sédiment, des impacts d'aménagements), leur utilisation pour l'étude de la dynamique de structures morphologiques telles que les bancs ou les dunes demeure limitée.

Ici, on étudie la dynamique des bancs de sable avec un modèle numérique 2DH. Les analyses de stabilité montrent qu'en terme de génération, les comportements de structures soumises à un courant alternatif ou stationnaire sont similaires [5]. Ainsi, l'étude est réalisée pour le cas d'un courant stationnaire.

Tout d'abord, le modèle numérique morphodynamique est présenté. L'analyse de stabilité linéaire et les simulations numériques sont réalisées puis comparées en terme de propriétés de stabilité du fond. Ensuite on estime la hauteur de saturation d'un banc linéairement amplifié. Enfin, l'évolution temporelle de l'amplitude du banc est simulée jusqu'à la saturation, à l'aide d'une équation de Landau. Une comparaison avec des données de terrain est réalisée avant de conclure.

\section{Présentation du modèle morphodynamique 2DH}

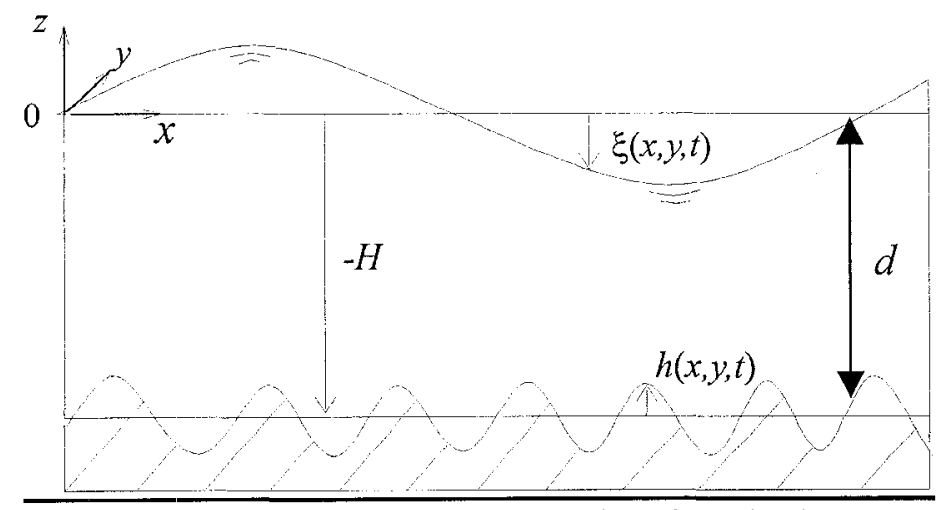

Figure 1 : Schéma du domaine de calcul.

Figure 1: Sketch of the model geometry.

Une granulométrie uniforme et un transport de sédiment saturé constituent les principales hypothèses du modèle d'évolution du fond. Ce modèle est basé sur l'équation de conservation du sédiment :

$$
\frac{\partial h}{\partial t}=-\vec{\nabla} \cdot \vec{S}_{b} \text { avec } \vec{S}_{b}=\alpha|\vec{u}|^{3}\{|\vec{u}||\vec{u}| \mid \lambda \vec{\nabla} h\}
$$

où $h$ est la cote du fond par rapport au fond non-perturbé $(z=-H)$ (Fig. 1), avec $\vec{S}_{b}$ le flux de sédiment calculé à l'aide de la formule de transport par charriage de Van Rijn [5]. $\alpha$ est un coefficient prenant en compte le coefficient de frottement et la porosité du fond, $\lambda$ le coefficient d'effet de pente (habituellement 1-3). Le logiciel Sisyphe [6] de la chaîne Telemac est utilisé pour résoudre l'équation (1).

Deux modèles sont utilisés pour le calcul des variables hydrodynamiques. Le 
premier est basé sur les équations de Saint-Venant stationnaires résolues par le logiciel Telemac2D [7]. Le second est un modèle hydrodynamique simplifié appelé «correction de continuité », directement intégré dans le logiciel Sisyphe. Le modèle morphodynamique s'appuie sur le couplage entre Telemac2D et Sisyphe. L'algorithme de couplage est le suivant. Tant que l'évolution du fond (calculée par Sisyphe à partir de l'hydrodynamique à l'instant courant) demeure inférieure à un seuil critique d'évolution du fond (fixé par l'utilisateur), les variables hydrodynamiques sont recalculées à partir de la nouvelle bathymétrie à l'aide du modèle hydrodynamique simplifié. Dès que ce seuil est atteint, l'hydrodynamique est réactualisée en résolvant les équations de Saint-Venant. Pour plus de détails sur le modèle, voir [8].

\section{Génération de bancs de sable}

Les taux de croissance et les vitesses de phase obtenus avec le modèle morphodynamique sont comparés à ceux obtenus par analyse de stabilité linéaire dans le cas d'une perturbation infinitésimale soumise à un courant quasistationnaire $\vec{u}=(U, 0)$.

\subsection{Résultats de l'analyse de stabilité linéaire}

Dans une analyse de stabilité linéaire, $\widetilde{h}_{1}$, la transformée de Fourier de l'amplitude adimensionnelle de la perturbation bathymétrique, est régie par :

$$
\frac{\partial \tilde{h}_{1}}{\partial \tau}=\omega \widetilde{h_{1}}
$$

Avec $\omega$ la fréquence complexe (la partie réelle $\operatorname{Re}(\omega)$ indique l'amplification ou la décroissance). Dans [5], une analyse de stabilité linéaire du modèle morphodynamique présenté dans le paragraphe précédent a été réalisée pour le cas d'un courant quasi-stationnaire. Toutefois, le terme de frottement dans les équations de quantité de mouvement était de la forme $r \vec{u} / d$, avec $r$ le coefficient de frottement. Dans le modèle numérique que nous utilisons, le frottement est de la forme $r\|\vec{u}\| \vec{u} / d^{4 / 3}$. En suivant la méthodologie de [5], nous avons effectué une nouvelle analyse de stabilité en prenant en compte le terme de frottement utilisé dans les simulations. La fréquence complexe suivante est obtenue :

$$
\omega=C^{3}\left(\frac{1}{3} \frac{i \hat{r} k}{-\hat{r}\left(2 l^{2}-9 k^{2}\right)-3 k^{2}\left(3 k^{2}+l^{2}\right)}-\hat{\lambda}\left(k^{2}\right)+k^{2}\left(k^{2}+l^{2}\right)\right)
$$

$\tau$ est l'échelle de temps morphologique, $\hat{r}$ le coefficient de frottement adimensionnel, $\hat{\lambda}$ le coefficient d'effet de pente adimensionnel, et $\vec{K}=(k, l)$ le vecteur d'onde adimensionnel (voir [5] pour la définition des grandeurs adimensionnelles). La vitesse de phase $\vec{C}_{w}$ est égale à $\operatorname{Im}(\omega) \vec{K} /\|\vec{K}\|^{2}$.

\subsection{Résultats du modèle numérique}


Des simulations numériques de l'évolution bathymétrique de perturbations d'amplitude infinitésimale $\left(1.67 \cdot 10^{-3}\right.$ de la hauteur d'eau $\left.H=30 \mathrm{~m}\right)$ et monochromatiques sont réalisées (chaque point dans l'espace spectral, Fig. 3, correspond à une simulation sur un an). Dans les simulations, les valeurs des coefficients adimensionnels sont : $\hat{r}=0.6 ; \hat{\lambda}=8.4 \cdot 10^{-3} ; \hat{\alpha}=5 \cdot 10^{-7} ; C=1$; pour une vitesse $U=1 \mathrm{~m} / \mathrm{s}$. Après chaque simulation, la fréquence complexe $\omega$ est calculée à partir des résultats obtenus pour $\mathrm{t}=1$ an et l'instant initial.

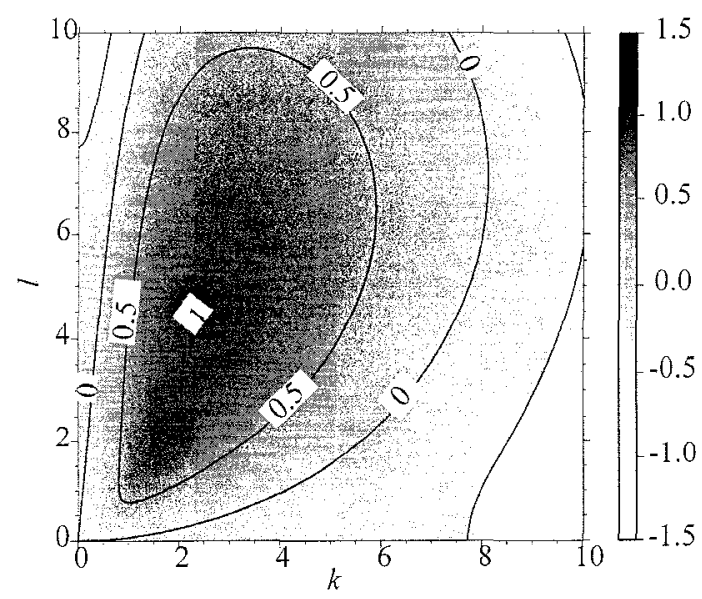

(a)

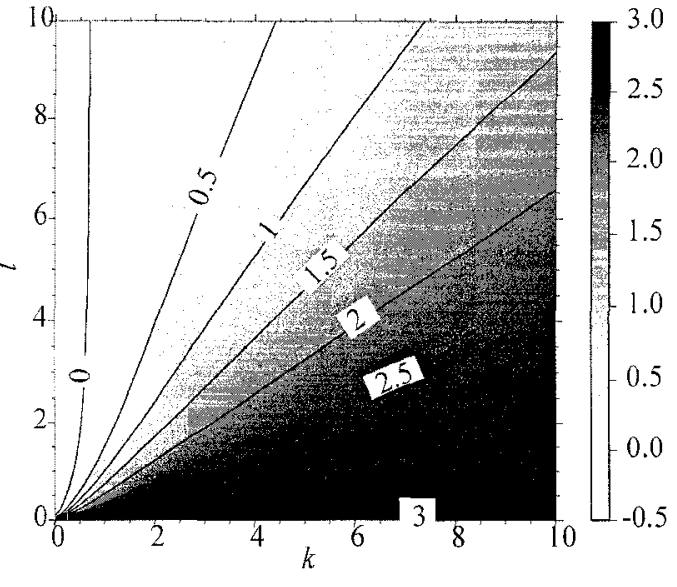

(b)

Figure 2 : Solution théorique. (a) : taux de croissance, (b) : vitesse de phase. Figure 2 : Theoretical solution. (a) : growth rate, (b) : phase celerity.

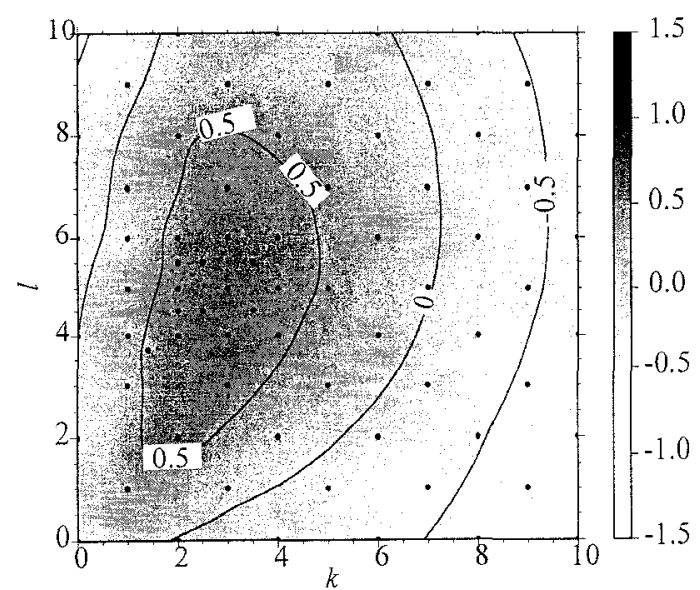

(a)

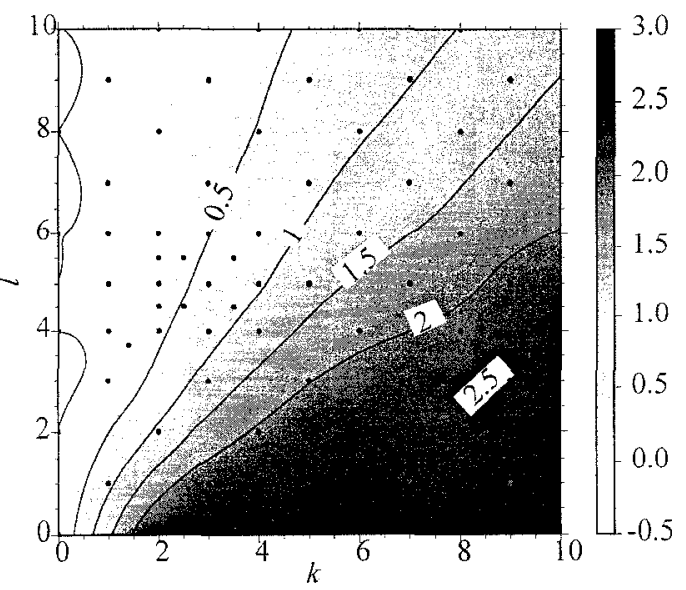

(b)

Figure 3 : Résultats du modèle. (a) : taux de croissance, (b) : vitesse de phase.

Figure 3 : Model results. (a) : growth rate, (b) : phase celerity.

Les figure $2 \mathrm{a}$ et $3 \mathrm{a}$ (taux de croissance) montrent une bonne adéquation du modèle numérique aux résultats théoriques. Il existe une zone de modes amplifiés, et les petites longueurs d'onde, i.e. les dunes et rides, sont amorties. D'après l'analyse de stabilité, le mode le plus amplifié est $(\mathrm{k}, 1)=(2.4,3.9)$ (banc de $9800 \mathrm{~m}$ de 
longueur d'onde, incliné de $32^{\circ}$ par rapport au courant incident). Des résultats numériques, on déduit le mode le plus amplifié $(\mathrm{k}, \mathrm{l})=(2.5,3.9)$. Donc, concernant le mode le plus amplifié, le modèle donne des résultats très satisfaisants, les différences étant dues à l'échantillonnage dans le plan spectral. La sousestimation du taux de croissance par le modèle peut avoir différentes origines dont la plus probable est la diffusion numérique inhérente aux schémas numériques de résolution du système.

La vitesse de phase (Fig. $2 b$ et $3 b$ ) montre que les deux approches fournissent des résultats très proches et prédisent une propagation anisotropique et non-dispersive des perturbations bathymétriques de faible amplitude (les perturbations ayant la même direction de propagation, perpendiculaire à leur crête, ont la même vitesse de phase).

\section{Comportement des bancs de sable à amplitude finie}

En théorie, le modèle morphodynamique permet de calculer l'évolution longterme d'une perturbation infinitésimale. Cependant, les coûts de calcul rendent ceci difficile. Une autre méthode est alors adoptée : le modèle numérique est utilisé pour déterminer la hauteur de saturation de la perturbation, puis les résultats du modèle sont utilisés pour calculer les coefficients d'une équation d'amplitude permettant de calculer l'évolution temporelle d'une perturbation jusqu'à sa saturation. Ici, l'étude est réalisée sur le mode $(k, l)=(1.4,3.7)$ qui correspond à un banc de longueur d'onde $11300 \mathrm{~m}$. Ce mode est celui le plus amplifié dans l'analyse de stabilité [5].

\subsection{Hauteur de saturation}

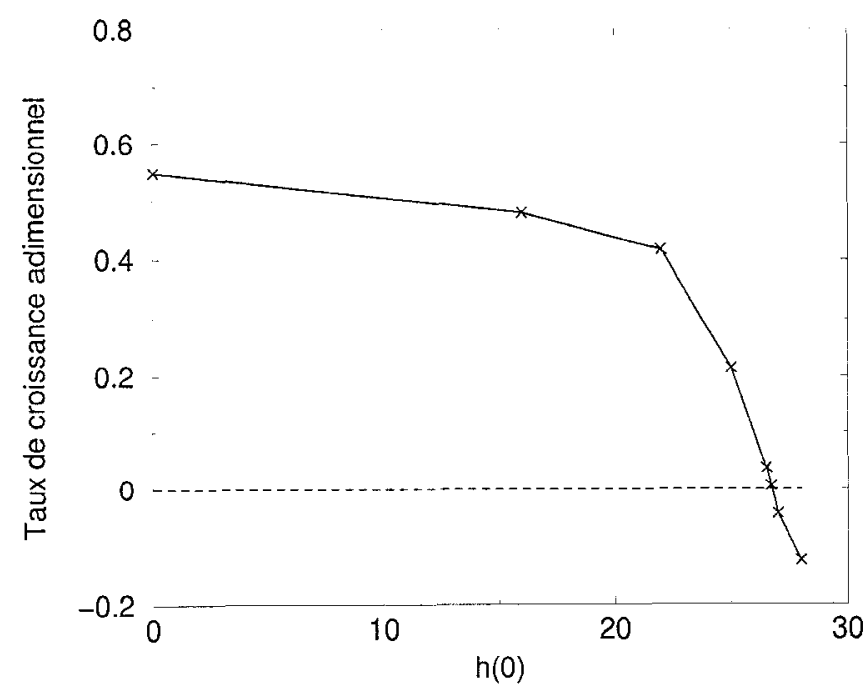

Figure 4: Taux de croissance $\operatorname{Re}(\omega)$ en fonction de l'amplitude initiale du banc.

Figure 4: Growth rate $\operatorname{Re}(\omega)$ versus the initial amplitude of the bank. 
La hauteur de saturation du banc est estimée en utilisant une méthode indirecte : plusieurs simulations sont réalisées pour des bancs ayant le même vecteur d'onde, mais avec des amplitudes initiales $h(0)$ différentes. On observe que le taux de croissance est une fonction décroissante de la hauteur initiale du banc (Fig. 4) et devient négatif pour des amplitudes de bancs supérieures à $26.74 \mathrm{~m}$. L'amplitude de saturation est donc estimée à $26.74 \mathrm{~m}$, ce qui correspond à un banc de $53.4 \mathrm{~m}$ de haut dans $56.7 \mathrm{~m}$ de fond.

L'analyse des termes d'effet de pente et de vitesse montre que les termes de vitesses (Eq. 1) sont prépondérants par rapport aux termes d'effet de pente et donc que l'effet de pente n'est pas le processus responsable de la saturation, ce qui est cohérent avec les faibles pentes des bancs observés in-situ [9].

\subsection{Evolution temporelle de l'amplitude du banc : de la génération à la saturation}

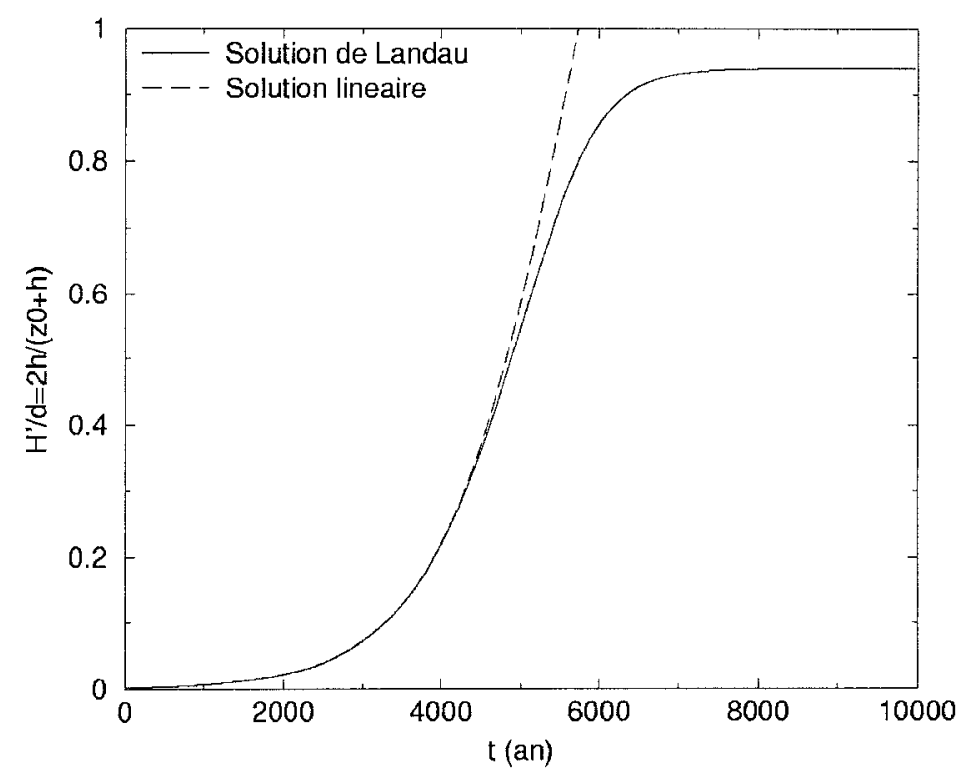

Figure 5: Evolution temporelle de la hauteur relative du banc.

Figure 5: Temporal evolution of the relative height of the sandbank.

L'équation (2) n'est valable que pour des structures d'amplitude infinitésimale. Pour étudier le comportement à amplitude finie, on utilise alors l'équation de Landau à coefficients réels qui permet une croissance exponentielle, mais aussi la saturation. Cette équation a prouvé son utilité pour l'étude des dunes [10].

$$
\frac{\partial \widetilde{h}_{1} \mid}{\partial \tau}=\operatorname{Re}(\omega)\left|\widetilde{h}_{1}\right|-\psi\left|\widetilde{h}_{1}\right|^{3}
$$

$\psi=\operatorname{Re}(\omega) /\left|\widetilde{h}_{1}(\infty)\right|^{2}$ étant le coefficient de Landau réel.

En utilisant les résultats du modèle numérique, on déduit un taux de croissance $\operatorname{Re}(\omega)=0.546$, et le coefficient de Landau $\psi=0.688$. 
L'évolution temporelle de la hauteur du banc est alors calculée à partir de l'équation 4 (Fig. 5). Après 8000 ans, le banc atteint sa hauteur de saturation qui est ici de $94 \%$ de la hauteur d'eau.

\subsection{Comparaison avec des bancs réels}

Les résultats ont été obtenus pour des paramètres correspondant aux caractéristiques de la Mer du Nord, dans le cas d'un courant unidirectionnel de 1 $\mathrm{m} / \mathrm{s}$, et pour un fond sableux. Le mode le plus amplifié est alors un banc incliné de $21^{\circ}$ par rapport au courant. Comme données in-situ, nous choisissons donc des bancs de la Manche et de la Mer du Nord inclinés d'environ $20^{\circ}$ par rapport au courant, reposant dans des zones sableuses et où le courant est fortement alternatif et d'intensité minimum de $1 \mathrm{~m} / \mathrm{s}$ (Tab. 1).

Les cartes de niveau des mers [11] montrent que les zones où reposent les bancs (Tab. 1) ont été inondées entre 8700 et 7800 avant l'époque actuelle. L'âge maximum des bancs est donc de 8700 ans.

Le temps de formation du banc obtenu avec le modèle numérique est de 8000 ans. Ce résultat est donc cohérent avec les données de terrain.

En outre, la hauteur relative des bancs est de l'ordre de $80 \%$ de la hauteur de d'eau alors que le modèle donne une hauteur de saturation de $94 \%$, i.e. un banc de $53.5 \mathrm{~m}$ dans $56.7 \mathrm{~m}$ de hauteur d'eau. Le modèle surestime donc la hauteur de saturation. Les explications peuvent être diverses: le modèle ne prend pas en compte l'action de la houle, les simulations ont été effectuées pour un courant stationnaire, le banc est supposé de forme sinusoïdale.

\begin{tabular}{|l|c|c|c|}
\hline Banc & $\begin{array}{c}\text { Hauteur d'eau } \\
(\boldsymbol{m})\end{array}$ & $\begin{array}{c}\text { Hauteur de banc } \\
(\mathbf{2 h}(\boldsymbol{m}))\end{array}$ & $\mathbf{2} \boldsymbol{h} / \boldsymbol{d}$ \\
\hline Schole (Manche) & 50 & 40 & 0.8 \\
\hline Sandettie (Mer du Nord) & 30 & 25 & 0.83 \\
\hline Southfalls (Mer du Nord) & 30 & 25 & 0.83 \\
\hline Casquet sse (Manche) & 36 & 29 & 0.805 \\
\hline
\end{tabular}

Table 1 : Bancs de sable tidaux.

Table 1 : Tidal sandbanks.

\section{Conclusion}

Le modèle numérique utilisé a prouvé sa capacité à reproduire correctement les propriétés de stabilité du fond. La hauteur de saturation de bancs tels que ceux de la Mer du Nord a été estimée égale à $94 \%$ de la hauteur d'eau, ce qui surestime les hauteurs de saturation issues des données de terrain. Enfin, l'évolution temporelle de l'amplitude d'un banc est simulée, de sa génération à sa saturation. Le temps nécessaire pour que le banc atteigne sa hauteur de saturation semble cohérent avec l'âge estimé des bancs réels. La prochaine étape sera de vérifier les mêmes propriétés de stabilité du fond pour les dunes, afin de pouvoir simuler l'évolution 
de dunes d'amplitude finie.

Remerciements. Les auteurs remercient S. Hulscher de l'université de Twente et V. Guimet de CS, pour leurs remarques, T. Garlan et A . Ehrhold du SHOM, pour avoir fourni les données, enfin, le SHOM et le CNRS, pour leur support financier.

\section{Références}

1. De Moor G. Maintenance on the Flemish Banks, in The Quaternary and Tertiary Geology of the Southern Bight, North Sea, edited by J.P. Henriet and G. de Moor, pp. 185-216, 1989.

2. Le Bot S., Idier D., Garlan T., Trentesaux A. and Astruc D. Dune dynamics: from field measurements to numerical modelling. Application to bathymetric survey frequency in the Calais-Dover Strait. pp. 101-108, Lille, "Marine Sandwave Dynamics, International Workshop », 2000.

3. Hulscher S.J.M.H. Tidal-induced large-scale regular bed form patterns in a three-dimensional shallow water model, J. Geophys. Res., vol. 101, C9, pp. 20,727-20,744, 1996.

4. Komarova N.L. and Newell A. Nonlinear dynamics of sandbanks and sandwaves. J. Fluid Mech., vol. 415, pp. 285-321, 2000.

5. Fluit C.C.J.M. and Hulscher S.J.M.H. Morphological response to a gas-mining induced bed depression in the North Sea. A paraitre dans J. Geophys. Res., 2002.

6. Peltier E. and Latteux B. TSEF Version 3.1 - Principle Note. EDF report HE42/95.060, 1995.

7. Hervouet J.M. and Van Haren L. TELEMAC-2D Version 3.0 - Principle Note. EDF Report HE-43/94/052/A, 1994.

8. Idier D. and Astruc D. Numerical modelling of large scale rhythmic bedforms in shallow-water. River, Coastal and Estuarine Morphodynamics Proceeding, pp. 565-574, Obihiro, Japan 2001.

9. Stride, A.H., Offshore tidal sands - Processes and Deposites, 222 pp., Chapman and Hall Ltd, 1982.

10.Knaapen M.AF., Hulscher S. and Scholl O. Can we predict the growth of sand waves? Hindcast of a field experiment in the Bisanseto Sea, Japan. Proceedings Coastal Engineering 2000, vol. 3, ASCE, pp. 2661-2671, 2001.

11. Jelgersma S., Sea-level changes in the North Sea basin. In Oele, E., Schuttenhelm, R.T.E. and A.J. Wiggers (Ed.), The quaternary History of the North Sea, Acta Univ. Upps., Uppsala, 233-248, 1979. 